\title{
Medical Emergencies in Oral and Maxillofacial Surgical Practice
}

\author{
Nallamilli V. S. Sekhar Reddy
}

\subsection{Introduction}

Oral and Maxillofacial Surgery has evolved over the last few decades. Although it is now practiced as a full-fledged hospital-based specialty, a significant amount of work is still carried out under local anesthesia; predisposing toward a medical emergency precipitated particularly by stress. Oral and Maxillofacial surgeons thus deal with the medical emergencies in an office or hospital setting on a regular basis.

Emergency team response in most countries is prompt. The published guidelines from these countries reflect this in the advice for the general practitioner; doing the essential minimum toward the sustenance of life in case of a medical emergency and to prioritize escalation of call for help to the emergency team. However, in some parts of the world, the response time of the emergency team is expected to be comparatively longer, due to various policy issues. The chapter considers these special circumstances, to suggest some additional measures toward the management of the emergency, while waiting for the arrival of the emergency team.

Also, the general advice in the management of a medical emergency during an outpatient procedure in a clinic is aimed at general practitioners, who may not necessarily be exposed to training in clinical skills like the placement of an intravenous cannula. Oral and Maxillofacial surgeons are expected to be well versed with this life-saving simple clinical skill and the protocols discussed here take this into consideration.

The management of emergency should follow the universally accepted ABCDE approach [1], which helps the clinician to follow a systematic approach in an emergency situation where the most life-threatening condition is assessed and is given the top priority. Lack of airway kills the patient first, then the lack of ability to breathe followed by the absence of circulation. ABCDE approach incorporates:

N. V. S. Sekhar Reddy $(\bowtie)$

Panineeya Institute of Dental Sciences and Research Centre,

Hyderabad, Telangana, India
A: Airway
B: Breathing
C: Circulation
D: Disability (refers to neurological function)
E: Exposure

Medical Emergencies that are life threatening and need immediate remedial measures on an emergency basis are discussed here:
1. Acute Asthmatic Attack
2. Adrenal Crisis
3. Airway Obstruction
4. Anaphylaxis
5. Chest Pain
6. Cardiac Arrest
7. Hypoglycemia
8. Tonic-Clonic Seizure
9. Syncope.

\subsection{Acute Asthmatic Attack}

Stress, Anxiety, and Infection can precipitate an asthmatic attack in individuals who are prone. Unfortunately, all three of them can be part of OMFS practice.

\section{Box 4.1}

Note: Asthma that is part of a generalized anaphylactic reaction needs Adrenaline via intramuscular route.

Signs and Symptoms:

- Difficulty in breathing

- Cough

- Wheezing

- Use of accessory muscles of respiration

- Tightness of chest 
- Rapid breathing and Tachycardia

- Pale and anxious-looking face

\section{Management:}

- Keep the patient in a sitting position.

- Give two puffs of Salbutamol (100 $\mu \mathrm{g} / \mathrm{puff})$ inhaler [2, 3].

- If no rapid response, give further puffs through a spacer device.

- Consider oxygen supplementation.

- If no improvement, consider urgent transfer to a medical center.

\subsubsection{Life-Threatening Acute Asthma}

Asthmatic attack can sometimes be severe and may fail to respond to standard bronchodilator therapy. This can be fatal if no proper treatment is given. When an asthmatic attack failed to respond to the treatment and getting worse, the patient will need urgent transfer to appropriate medical center.

Signs and symptoms of severe life-threatening asthma:

- Silent chest, poor respiratory effort, cyanosis

- $\mathrm{SPO}_{2}<92 \%$

- Exhaustion

- Altered consciousness

- Weak or irregular pulse

Treatment:

- Urgent transfer to hospital is needed.

- Oxygen supplementation.

- Salbutamol $100 \mu \mathrm{g} /$ puff; give four puffs through spacer device, followed by two puffs every 2 min according to response, up to a maximum of ten puffs or if there is nebulizer available, give salbutamol $5 \mathrm{mg}$ [4].

- Hydrocortisone: $100 \mathrm{mg}$ IV, or Prednisolone: 40-50 mg Orally [4].

- For children 5 years and above, use Oral Prednisolone 30-40 mg.

- For children less than 5 years, give salbutamol inhalation up to a maximum of ten puffs while awaiting transfer to an appropriate medical facility [2].

\subsection{Acute Adrenal Insufficiency/Adrenal Crisis/Steroid Crisis/Addisonian Crisis}

Sudden and severe hypotension in patients who cannot mount a normal stress-induced cortisol response. The stress can be physiological or psychological. Adrenal crisis can be seen in patients who were taking glucocorticoids regularly or who have taken glucocorticoids for a considerable period of time in the past, patients with Addison's disease, hypopituitarism, or in other conditions associated with decreased adrenocorticotropic hormone (ACTH) production.
Box 4.2

Oxygen-Supports Combustion

Keep cylinders away from open flames and heat sources

Oxygen cylinder should be stored in a wellventilated area

Do not oil, grease, or lubricate cylinder controls.

\section{Signs and Symptoms:}

- Severe hypotension, which can manifest as weakness, confusion, dizziness, drowsiness, loss of consciousness.

- Nausea, vomiting.

- Seizure may occur following deep faint.

\section{Treatment:}

- Lay patient flat.

- Give high flow oxygen.

- Arrange for urgent transfer to a medical center.

- Establish Intravenous access.

- Administer Hydrocortisone sodium succinate by IV. Dosage given below [5, 6]:

- Children from age 2 to 11 years, $2-4 \mathrm{mg} / \mathrm{kg}$ body weight.

- Above 11 years: give adult dose.

- Adult dose: $100 \mathrm{mg}$ by IV.

- If unable to secure IV access, give the same dose by IM route.

- Start IV infusion of 0.9\% Normal Saline.

\subsection{Airway Obstruction}

As the mouth is the primary area of our specialty, clinicians may have to deal with acute airway obstruction. As with everything else, prevention is the most important thing.

\section{Signs and Symptoms:}

- Distressed patient.

- Abnormal noises like gurgling, crowing, snoring.

- Forced inspiratory efforts.

- No sounds at all in complete airway obstruction

- Various degrees of cyanosis leading to loss of consciousness.

Treatment [7]:

- Encourage the patient to cough.

- If an object could be visualized, retrieve it with an appropriate instrument.

- If obstruction is caused by fluids, use high-volume suction.

- Back slaps: up to five back blows between the scapulae while the patient is supported leaning forward.

- Abdominal thrusts: (Heimlich maneuver) up to five abdominal thrusts by standing behind the patient. 
- If obstruction fails to relieve, continue with five back blows alternating with abdominal thrusts.

- If these measures fail, the patient will lose consciousness. Lay the patient down on floor and start with CPR.

- Start with chest compressions even if there is pulse, as chest compressions can help relieving the obstruction.

- If there is partial obstruction, which could not be relieved by the foregoing means, arrange for emergency transfer to appropriate medical care.

- Patient may need oxygen supplementation.

- In patients with a completely obstructed airway, where the obstruction could not be relieved with these measures, where there are no facilities or skill to use a laryngoscope, emergency invasive procedures like jet insufflation or cricothyroidotomy may be needed as life-saving measures.

- Jet Insufflation involves placing a wide bore cannula (1214 gauge) through cricothyroid membrane and connect this to a high flow oxygen source.

- It is advised to read further about Jet insufflation and Cricothyroidotomy as the full detailed description is beyond the scope of this textbook.

\section{Box 4.3}

Aspiration of small objects like burs, endodontic files, and reamers may not cause immediate lifethreatening airway obstruction, but retrieval of these rather small and prickly instruments from lungs is a very difficult task needing bronchoscopy and can result in serious lung infections and damage. Prevention takes priority.

\subsection{Anaphylaxis}

Sudden onset, severe form of life-threatening allergic reaction, which can be seen following exposure to an allergen. Remember topical anesthetic gels, chlorhexidine mouth wash, and even contact with latex gloves can trigger anaphylaxis in individuals who are sensitive [8].

Prompt recognition and immediate response are key to success.

\section{Signs and Symptoms:}

- Face—red and hot.

- Generalized skin rash with itching.

- Various degrees of breathing difficulties manifested as stridor, wheezing, or hoarseness.

- Pulse may initially be rapid but can gradually become weak or impalpable.

- Hypotension can progress to cardiac arrest.

\section{Treatment:}

- Make sure airway is clear.

- Administer Adrenaline [9].
- Position the patient in supine position unless the patient prefers to sit up due to breathing difficulties.

- Make arrangements for emergency transfer to appropriate medical center.

- While waiting for transfer [10],

- Oxygen supplementation at $15 \mathrm{~L} / \mathrm{min}$.

- Keep assessing the airway and provide necessary support.

- Chlorpheniramine maleate IM or IV.

- Hydrocortisone sodium succinate IM or IV.

- Establish Intravenous access.

- Ringer's lactate solution or $0.9 \%$ Normal saline to support circulation.

- Reassess the need for further adrenaline.

- Start CPR in case of cardiac arrest.

Box 4.4

Adrenaline is to be administered if there is severe respiratory distress or when there are signs of shock.

Box 4.5

Adrenaline - Life-saving drug for Anaphylaxis

Repeat after $5 \mathrm{~min}$ if not getting better

Supply: $1 \mathrm{~mL}$ ampoule containing $1 \mathrm{mg}$ adrenaline

in 1:1000 concentration

Site: I.M Anterolateral thigh

Dosage:

Adult: $500 \mu \mathrm{g}$ IM $0.5 \mathrm{~mL}$

Child More than 12 years: $500 \mu \mathrm{g}$ IM $0.5 \mathrm{~mL}$

Child 6-12 years: $300 \mu \mathrm{g}$ IM $0.3 \mathrm{~mL}$

Less than 6 years: $150 \mu \mathrm{g}$ IM $0.15 \mathrm{~mL}$

\section{Box 4.6}

\section{Second-line drugs:}

Hydrocortisone sodium succinate: $100 \mathrm{mg}$

Route: IM or Slow IV

12 years and adults: $200 \mathrm{mg} \mathrm{IM} / \mathrm{IV}$

6-12 years: $100 \mathrm{mg} \mathrm{IM/IV}$

6 months to 6 years: $50 \mathrm{mg} \mathrm{IM/IV}$

Chlorpheniramine Maleate

Supply: Chlorpheniramine Maleate $10 \mathrm{mg} / 1 \mathrm{~mL}$

Ampoule

Route: IM or IV

12 years and adults: $10 \mathrm{mg}$ IM/IV

6-12 years: $5 \mathrm{mg} \mathrm{IM/IV}$

6 months to 6 years: $2.5 \mathrm{mg} \mathrm{IM/IV}$ 


\subsection{Chest Pain}

Experiencing chest pain in an outpatient office can be a frightening experience for both the patient and to the attending clinician. Thorough understanding of the pathophysiology of these acute cardiac events is very important for the clinician to stay composed and initiate appropriate measures to prevent mortality. Myocardial infarction may not always have the typical features of chest pain and especially diabetic patients may not have pain. Hence, a high index of suspicion is what is required.

Causes of chest pain:

- Angina

- Myocardial Infarction

- Hyperventilation

Hyperventilation is associated with anxiety and often a young person who is quite frightened of the intended treatment, the breathing gets very heavy resulting in decreased carbon dioxide levels in the blood.

\subsubsection{Angina}

Transient decrease of oxygen supply to cardiac muscle precipitates pain.

Precipitating factors:

Anxiety, stress, exertion, strong emotions, heavy meal, and extreme weather conditions.

\section{Signs and Symptoms:}

If you are looking for typical symptoms of chest pain, you may miss some cases. List of all possible presentations being:

- Pain, heaviness, or tightness in the chest.

- Pain in the neck, throat, arms, stomach, and back.

- Pain can be associated with light-headedness, nausea, shortness of breath, and sweating.

\section{Management:}

- Stop the procedure and reassure the patient.

- Clear the mouth from any foreign bodies.

- Let the patient attain a comfortable position (may not like to lie flat).

- Administer Glyceryl trinitrate sublingual spray or Glyceryl trinitrate sublingual tablets [11].

- Oxygen supplementation.

- Check for any irregularities of pulse.

Simple angina should resolve very quickly with rest alone or with sublingual glyceryl trinitrate spray or tablets. In simple angina, the cardiac output is not impaired, and pulse should be regular. In a patient with chest pain, having an irregular pulse, suspect MI.

\subsubsection{Myocardial Infarction (Heart Attack)}

Progressive/Sudden ischemia of the cardiac muscle leads to necrosis of cardiac muscle. This can cause cardiac arrest.

\section{Signs and Symptoms:}

- Same as angina but can be more severe in intensity and lasts long.

- Pain in the center of the chest.

- Pain radiates to neck, arms, jaw, back, or stomach.

- Pain associated with sweating, vomiting, shortness of breath, lightheadedness, or dizziness.

- Chest discomfort associated with a feeling of weakness and unwell.

- Chest pain associated with an irregular pulse.

- Chest pain fails to relieve with two doses of Nitroglycerin sublingual Spray.

- Chest pain getting progressively worse.

- Patient with previous experience of angina, now experiencing an unusual type of chest discomfort.

- Weak pulse.

- Falling Blood pressure.

- Skin-pale and clammy.

\section{Management:}

- The moment MI is suspected, arrange for an emergency transfer of the patient to an appropriate medical facility.

- Let the patient attain the most comfortable position. The patient may like to sit up to avoid venous congestion on the heart and lungs.

- Administer soluble Aspirin, 300 mg [12]. Chew and swallow for rapid action.

- By this time, the patient should have received at least two doses of sublingual Glyceryl trinitrate spray or tablets [12], this may need a repeating.

- While waiting for transfer, the following measures can be done, but do not delay transfer, early expert care improves outcome.

- Establish IV access.

- Give antiemetic Metoclopramide hydrochloride by IV [13].

Adult $>60 \mathrm{~kg}$, dose $10 \mathrm{mg}$

Small-sized Adult $<60 \mathrm{~kg}$, dose $5 \mathrm{mg}$

- Administer analgesia - Paracetamol by slow IV (Morphine, diamorphine 7 fentanyl, are not easily available in India) [13]. 
Adult $>50 \mathrm{~kg}$, dose $1000 \mathrm{mg}$ in $100 \mathrm{~mL}$

Small-sized Adult $<50 \mathrm{~kg}$, dose $750 \mathrm{mg}$ in $75 \mathrm{~mL}$

When no IV access, alternatively, give Oral Paracetamol $650 \mathrm{mg}$

- Do not routinely administer oxygen. Supplemental oxygen only when hypoxic.

- Patients where consciousness level deteriorates.

- Watch for signs of cardiac arrest.

- Start CPR, if cardiac arrest occurs.

\section{Box 4.7}

Drugs for MI:

Glyceryl Trinitrate Aerosol Spray: $400 \mu \mathrm{g} /$ metered dose

Glyceryl Trinitrate: $300-\mu \mathrm{g}$ tablets

Soluble Aspirin: 300-mg tablets

Metoclopromide: 10-mg solution for IV

Paracetamol: 1000-mg/100-mL solution for IV

Paracetamol: 500-mg tablets

Paracetamol $650 \mathrm{mg}$ tablets
- Not breathing normally (agonal breathing/gasping) or absent breathing

- Some people may have a seizure when cardiac arrest occurs as a result of severe cerebral hypoxia.

\section{Box 4.8}

Chest pain in a patient with previous history of stable angina: Urgent transfer to appropriate medical facility is to be done, if the patient feels the pain is unusual or if the pain doesn't resolve with rest and Sublingual Glyceryl Trinitrate.

\section{Box 4.9}

Do not waste time, for example, trying to find a vein while the ambulance is waiting! Antiplatelet medication (aspirin) within few minutes of suspecting MI and early thrombolysis by the Physicians can significantly reduce the mortality associated.

\section{Management:}

- Call for help and ask to bring defibrillator and emergency drug kit.

- Second person can call for ambulance.

- Start CPR [14]

- CPR can be executed in the dental chair.

- Start with chest compressions.

- Second person checks airway, gets ready for ventilations, preferably using a bag valve mask.

- Give 30 chest compressions.

- Give two ventilations.

- Carry on CPR at 30:2 until expert help or AED arrives.

- Stop CPR when patient shows signs of life. CPR with AED:

- Continue with CPR while the pads of AED are being attached.

- Stop CPR and do not touch victim while the AED starts analyzing the rhythm [15].

- Nonshockable rhythm observed by AED—continue CPR until signs of life or AED starts analyzing rhythm again [16].

- Shock advised by AED-Make sure no one touches the patient directly or indirectly-Deliver the shock.

- Continue CPR at 30:2 while the pads remain attached.

- After 2 min, AED will start reanalyzing the rhythm and the cycle to be continued until the patient shows signs of life.

- Unconscious

- Unresponsive 
Fig. 4.1 Chain of survival

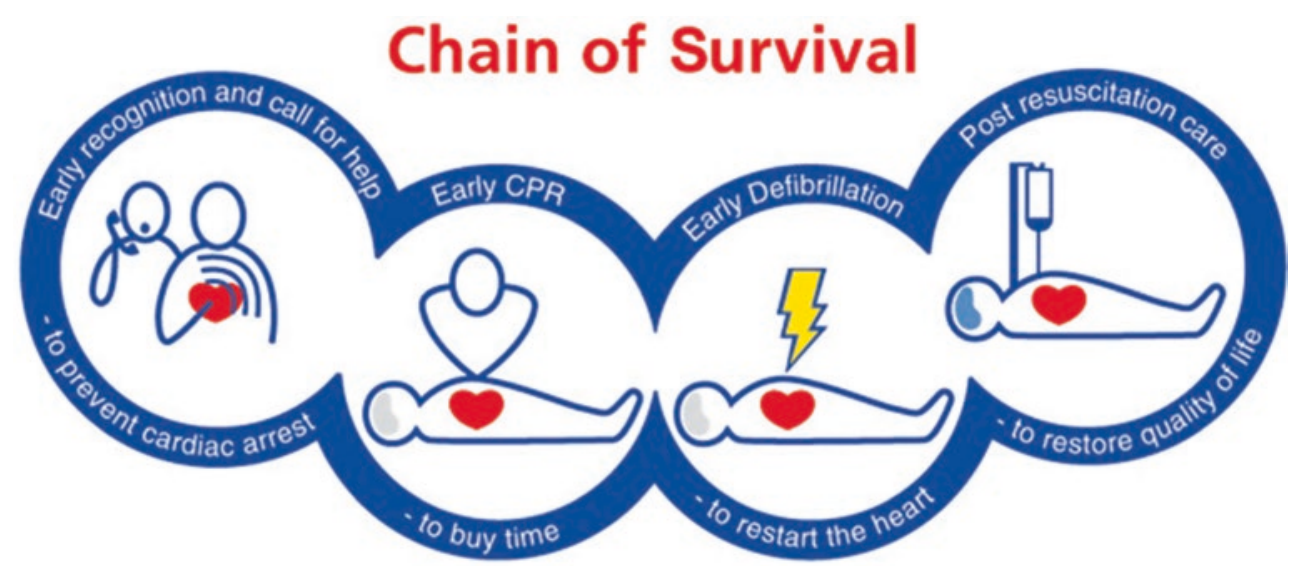

- Once the patient regains consciousness, put the patient in recovery position and maintain saturations above $94 \%$, supplemental oxygen may be needed.

- The patient should receive appropriate post-resuscitation care and keep monitoring as chances of a second cardiac arrest is high in the immediate post-resuscitation period.

To improve overall outcome in cardiac arrest patients, it is necessary to follow these four important aspects of care:

1. Early recognition and call for help - do not waste time trying to ascertain if pulse is there or not. A patient who is unresponsive and not breathing should be considered in cardiac arrest unless until proven otherwise.

2. Early $C P R$ - the victim soon after cardiac arrest will have some oxygen in the blood and starting with chest compressions early will be extremely beneficial to the cells starved of oxygen.

3. Early Defibrillation: The chances of reversing a shockable rhythm diminish with the passage of time.

4. Post resuscitation care: All patients who have been successfully reversed from cardiac arrest should be transferred to an appropriate center with cardiac care facilities.

The four points are incorporated into this internationally recognized "chain of survival," which is given here (adapted from the Resuscitation Council UK; website: https://www. resus.org.uk/EasysiteWeb/getresource.axd?AssetID=3907\& type $=$ Full\&servicetype=Attachment) (Fig. 4.1):

\section{Box 4.10}

All healthcare workers should undergo proper training in CPR. Healthcare organizations throughout the world are now insisting on a CPR certificate. Such a training has to be done on a periodic basis so that the skills are retained. The reader is strongly advised to undergo training periodically and a valid certificate will prevent you from future litigation if an unfortunate event occurs to one of your patients.

\subsection{Hypoglycemia}

Glucose is essential for the effective functioning of neurons. When the glucose levels in blood drop to a very low level, brain function will get affected and if the glucose levels are not restored quickly, permanent neurologic damage can occur within 4-6 min. Hypoglycemia can occur in diabetic patients taking Insulin, who skipped meal, and is also the leading cause of loss of consciousness in children. Hypoglycemia occurs when blood glucose level falls below $70 \mathrm{mg} / \mathrm{dL}$.

\section{Signs and Symptoms:}

- Anxious, confused, irritable, or aggressive behavior.

- Sweating

- Nausea and vomiting

- Hungry

- Visual disturbances

- Convulsions

- Loss of consciousness.

\section{Management [17]:}

Management depends on consciousness level and the ability of the patient to take food orally without the risk of aspiration.

- Conscious patient:

- 10-20 g of glucose

$10 \mathrm{~g}$ of glucose in two teaspoons of granulated sugar Two teaspoons of Glucon-D powder contain $15 \mathrm{~g}$ of Glucose

- Can be repeated in 10-15 min

- Once the patient's condition improves, provide a carbohydrate snack or meal as appropriate to raise blood glucose level.

- Unconscious patient:

- Check if airway is clear-ABCDE approach

- Put patient in recovery position

- Oxygen supplementation at $15 \mathrm{~L} / \mathrm{min}$ 
- Give Glucagon by IM route into the anterolateral thigh

- If Glucagon is not available

Arrange for emergency transfer of patient

Give $100 \mathrm{~mL}$ of $10 \%$ dextrose by IV route

Severe cases of hypoglycemia need $50 \mathrm{~mL}$ of $50 \%$

Dextrose

Higher concentrations of dextrose are thicker solutions, need a larger bore cannula.

Once the patient regains consciousness, give oral glucose and carbohydrate as earlier.

\section{Box 4.11}

Glucagon either by IM or SC route

Dose: Adult-1 mg

Child under 8 years or less than $25 \mathrm{~kg}$ should be given $500 \mu \mathrm{g}(0.5 \mathrm{mg})$

Keep patient in lateral position to prevent risk of aspiration

\subsection{Grand Mal Seizures}

Enquire with patients about how frequently they get seizures and their compliance with medication. Patients with frequent seizure history or patients who are irregular with their medications are more likely to have a seizure while having dental procedures. Provide the treatment in a calm, stress-free environment, avoid any seizure-triggering factors.

\section{Signs and Symptoms:}

- Seizure activity may have a brief period of aura, where the patient can behave indifferently, spaced out, and detached.

- The patient will go rigid, lose consciousness, lose balance, and may cry-tonic (rigidity) phase.

- After a few seconds, sudden rhythmic jerking movements of the body lasting for up to $2 \mathrm{~min}$.

- Mouth goes rigid. Frothy saliva can be noticed.

- Cyanosis around the mouth can be noticed as breathing stops.

- Possible urination.

- Following the seizure, patient can go into varying periods of confused state and may fall asleep.

\section{Treatment [18]:}

- Prevent injury to patient

- Remove all the sharps away

- Create safe space around a fitting patient

- Provide cushions like a pillow or blanket

- Pay particular attention to head

- Do not restrain

- Do not attempt to insert any object between teeth

- Supplemental oxygen at $15 \mathrm{~L} / \mathrm{min}$

- Have a suction readily available, with a yankauer suction tube clear oral cavity of any fluids after seizure stops [19].

- Ascertain hypoglycemia as a possible cause by checking blood glucose level by a finger prick test. This should be done in a patient with no previous history of epilepsy. Hypoglycemia is common in children and in diabetic patients [19].

- Once the convulsions are stopped:

- Provide reassurance and empathy to the patient

- Explain what had happened

- Examine the mouth for any fluids and use suction

- Examine for any bleeding, lacerations of tongue secondary to biting

- Place the patient in a recovery position

- Examine for any other injuries

- Provide oxygen as required

- Stay with the patient until the patient is fully recovered

- Do not give anything orally until the patient is fully conscious, to avoid the risk of aspiration

- Do not attempt to arouse a patient who is sleeping after a convulsive episode

- Prolonged Convulsions beyond 5 min or repeated seizure activity without proper recovery constitutes-status epilepticus [20], which can be fatal and needs to be managed as:

- Make arrangements for urgent transfer to appropriate medical center

- Administer midazolam intravenously [21]

- Where IV access is not possible:

Rectal route can be used effectively, especially in children.

Buccal midazolam gel can be instilled into buccal vestibule with the help of a syringe with no needle.

Midazolam sprays, which can be used via nasal route, are slowly being available in India.

Buccal and nasal routes are increasingly being used and the reader is advised to look up for more up-todate information as suitable formulations are not routinely available in India.

\section{Box 4.12}

Midazolam Dosage:

I.V route: Above 12 years, $5 \mathrm{mg}$ slowly-patient may need encouragement to breathe after IV midazolam.

I.M route: Adult: $0.2 \mathrm{mg} / \mathrm{kg}$, Not to exceed $10 \mathrm{mg}$ in total.

Child: $0.1-0.2 \mathrm{mg} / \mathrm{kg}$, not to exceed $10 \mathrm{mg}$ in total Nasal Spray: Child: $0.2 \mathrm{mg} / \mathrm{kg}$ body weight (Max: $5 \mathrm{mg} /$ nares)

Rectal: Adult and children above 12 years: $10-20 \mathrm{mg}$, can be repeated once after 10-15 min if needed

Child 2-11 years: $5-10 \mathrm{mg}$, can be repeated once after $10-15 \mathrm{~min}$ 


\section{Box 4.13}

Remember generalized tonic-clonic seizures can be seen in:

- Epilepsy

- Head injury

- Hypoglycemia

- Hypoxia

- Alcohol withdrawal

- Drugs

- Local anesthesia overdose or Intravascular injection

- Febrile convulsions

\section{Box 4.14}

Midazolam is a benzodiazepine that carries the risk of respiratory depression. Patients need close monitoring after drug administration and may need respiratory support.

\section{Box 4.15}

Typical grand mal seizure, in a known epileptic patient, where the seizure has subsided within a couple of minutes, patient can be discharged with the carer, provided all the vital signs are with in normal range. Urgent hospital transfer is indicated in all other epileptic seizures.

\subsection{Syncope}

Energy demands of the brain are met primarily by oxidation of glucose. A regular and constant supply of glucose and oxygen is essential for brain function. Supply of both glucose and oxygen depends on effective perfusion of the brain. When brain perfusion decreases beyond a critical level, the patient loses consciousness and the balance gets affected and the patient falls.

\section{Signs and Symptoms:}

- Feeling unwell

- Nausea, Lightheadedness

- Blurred vision

- Pallor

- Sweaty, especially forehead

- Sudden loss of consciousness and collapse

- Seizure, if the patient is not positioned in a horizontal position quickly.

\section{Management [22]:}

- Clear airway and reassure

- Change position to a flat or legs elevated

- Let fresh air by opening a window or a fan

- Do not let people to crowd around

- Place cold towel on forehead

- Supplemental Oxygen can be given, but usually, if it's simple faint consciousness should recover very quickly.

- Once recovered, encourage the patient to have a sugary drink.

- Examine for injuries secondary to fall.

\subsection{Other Causes of Loss of Consciousness}

Although syncope is the most common cause of loss of consciousness, the other causes should also be borne in mind, as some of them can be quite serious, such as:

- Postural Hypotension

- Hyperventilation

- Epilepsy

- Adrenaline crisis

- Hypoglycemia

- Stroke

- Cardiac Arrest

\section{Postural Hypotension:}

- Loss of consciousness following a sudden change of posture from supine or sitting to standing upright.

- Usually in patients who are taking antihypertensives or elderly.

- Place them in a supine position and bring them to the upright position slowly. 


\section{Hyperventilation:}

Excessive breathing in an anxiety state or excessive crying resulting in drop of blood $\mathrm{CO}_{2}$ level.

- Feeling lightheaded, dizzy

- Chest discomfort to chest pain

- Muscle spasms, especially in hands and feet

- Tingling or numbness in the arms and in the perioral region

- Control the crying/breathing through suggestion

- Use a paper bag to rebreath exhaled air to build up $\mathrm{CO}_{2}$ levels

\subsection{Conclusion}

Preparedness for emergency is the key and when you are prepared, emergencies are easier to manage. The maxillofacial surgeon should be able to recognize these conditions and initiate appropriate management before professional emergency help is available. More often, maxillofacial surgeon is the lone clinician and is the sole responder in an emergency. It is prudent for the surgeon to know what to do and what not to do to get the best out of the worst scenario or situation.

It is imperative that the maxillofacial surgeons thoroughly understand the pathophysiology and extend their skills to recognize and manage expeditiously and effectively the emergencies that may arise. It is advisable that the contemporary clinicians update their knowledge and skills periodically with proper training courses.

Protocols given here are based on the currently available evidence, keeping in view of the local healthcare systems and access. As protocols are only suggestive, the clinician is strongly advised to use his or her own discretion when dealing with medical emergencies (Table 4.1; Flowchart 4.1).

Table 4.1 Causes of loss of consciousness and its age distribution

\begin{tabular}{|l|l|l|}
\hline Causes of unconsciousness & & \\
Child & Teen-mid 30 & Above 40 \\
\hline Hypoglycemia & Syncope & Cardiovascular causes \\
\hline Epilepsy & Hypoglycemia & \\
\hline Congenital heart disease & Epilepsy & \\
\end{tabular}

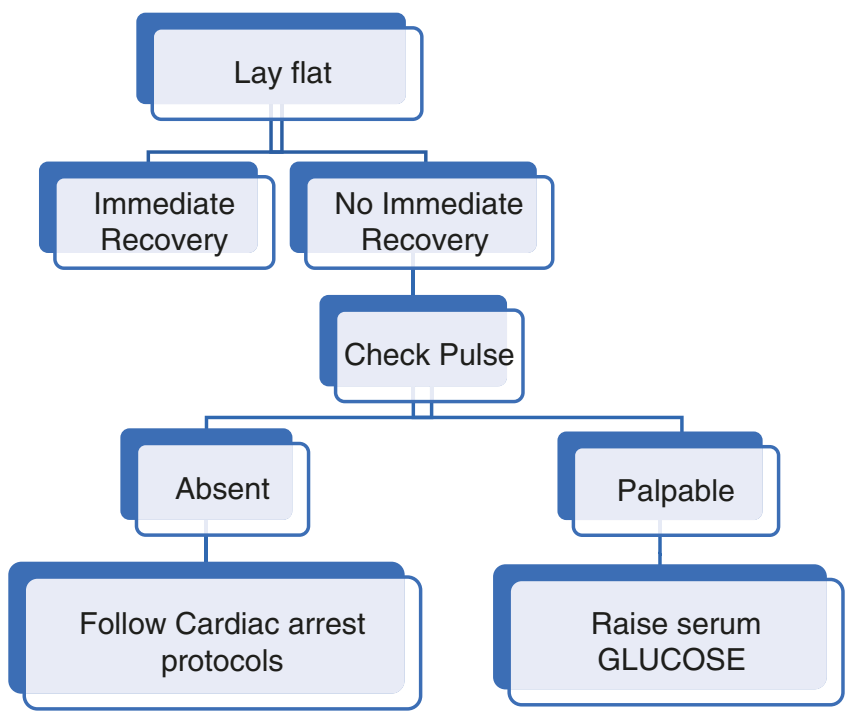

Flowchart 4.1 Management algorithm for a collapsed patient where the cause of collapse is not known

\section{References}

1. Thim T, Krarup NHV, et al. Initial asseement and treatment with the Airway, Breathing, Circulation, Disability, Exposure (ABCDE) approach. Int J Gen Med. 2012;5:117-21.

2. Saglani S, Fleming L, Sonnappa S, et al. Advances in the aetiology, management, and prevention of acute asthmatic attack in children. Lancet Child Adolesc Health. 2019;3(5):354-64.

3. Fergeson JE, Patel SS, Lockey RF. Acute asthma, prognosis, and treatment. J Allergy Clin Immunol. 2017;139(2):438-47.

4. Marsh B, Drake MG. Outpatient management of acute exacerbations of obstructive lung diseases. Med Clin N Am. 2017;101(3):537-51.

5. Hahner S. Acute adrenal crisis and mortality in adrenal insufficiency. Ann Endocrinol. 2018;79(3):164-6.

6. Paur THK, Stikkelbroeck NMMI, et al. Adrenal crisis: still a deadly event in the 21st century. Am J Med. 2016;129(3):339.

7. Schwartz A. Airway mangement for the oral surgery patient. Oral Maxillofac Surg Clin N Am. 2018;30(2):207-26.

8. Chapman J, Lalkhen AG. Anaphylaxis. Anaesth Intensive Care Med. 2017;18(1):16-21.

9. Young NH, Nimmo GR. Anaphylaxis. Medicine. 2017;45(3):157-62.

10. Moore LE, Kemp AM, Kemp SF. Recognition, treatment, and prevention of Anaphylaxis. Immunol Allergy Clin N Am. 2015;35(2):363-74.

11. Waller JR, Waller DG. Drugs for systemic hypertension and angina. Medicine. 2018;46(9):566-72.

12. Dym H, Barzani G, et al. Emergency drugs for the dental office. Dent Clin N Am. 2016;60(2):287-94. 
13. Herman WW, Ferguson HW. Dental care for patients with heart failure: an update. J Am Dent Assoc. 2010;141(7):845-53.

14. Yates EJ, Schmidbauer $S$, et al. Out of hospital cardiac arrest termination of resuscitation with ongoing CPR: an observational study. Resuscitation. 2018;130:21-7.

15. Fernando SM, Vaillancourt C, et al. Analysis of bystander CPR quality out of hospital cardiac arrest using data derived from AED. Resuscitation. 2018;18:138-43.

16. Pollack RA, Brown SP, et al. Bystander automated external defibrillator application in non-shockable out of patient cardiac arrest. Resuscitation. 2019;137:168-74.

17. Bansal N, Dhaliwal R, et al. Management of diabetes in elderly. Med Clin N Am. 2015;99(2):351-77.
18. Johnson EI. Seizures \& epilepsy. Med Clin N Am. 2019;103(2):309-24.

19. Thijs RD, Surges R, et al. Epilepsy in adults. Lancet. 2019;393(10172):689-701.

20. Paris M, Reddy U. Update on the management of status epilepticus. Anaesth Intensive Care Med. 2018;19(3):83-6.

21. Papavasiliou AS, Kotsalis C, et al. Intravenous midazolam in convulsive status epilepticus in children with pharmacoresistant epilepsy. Epilepsy Behav. 2009;14(4):661-4.

22. Numeroso F, Mossini G, et al. Emergency department management of patients with syncope according to the 2018 ESC guidelines. Int J Cardiol. 2019;283:119-21.

Open Access This chapter is licensed under the terms of the Creative Commons Attribution 4.0 International License (http://creativecommons. org/licenses/by/4.0/), which permits use, sharing, adaptation, distribution and reproduction in any medium or format, as long as you give appropriate credit to the original author(s) and the source, provide a link to the Creative Commons license and indicate if changes were made.

The images or other third party material in this chapter are included in the chapter's Creative Commons license, unless indicated otherwise in a credit line to the material. If material is not included in the chapter's Creative Commons license and your intended use is not permitted by statutory regulation or exceeds the permitted use, you will need to obtain permission directly from the copyright holder. 